\title{
Three-day regimen of oseltamivir
} for post-exposure prophylaxis of influenza in hospital wards: a study protocol for a prospective, multi-center, single-arm trial

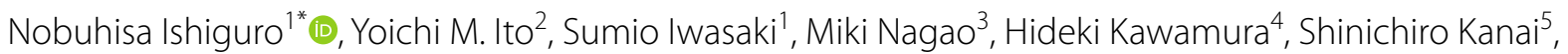
Yoko Nukui ${ }^{6}$, Koichi Tokuda ${ }^{7}$, Takayuki Miyara ${ }^{8}$, Hidetoshi Igari ${ }^{9}$, Koichi Yamada ${ }^{10}$, Hiroki Chikumi ${ }^{11}$, Chiaki Sano ${ }^{12}$, Ryuji Koike ${ }^{6}$, Tetsuya Yagi ${ }^{13}$ and Nobuo Murakami ${ }^{14}$ Japan Infection Prevention, Control Conference for National, Public University Hospitals

\begin{abstract}
Background: In a previous retrospective observational study, a 3-day regimen of oseltamivir as post-exposure prophylaxis (PEP) for preventing transmission of influenza in wards was shown to be comparable to 7- to 10-day regimens provided index cases were immediately separated from close contacts. In order to confirm the efficacy of a 3-day regimen, we started to conduct a prospective, multi-center, single-arm trial.

Methods: This study is a prospective, multi-center, single-arm study designed by the Sectional Meeting of Clinical Study, Japan Infection Prevention and Control Conference for National and Public University Hospitals. Index patients with influenza are prescribed a neuraminidase inhibitor and are discharged immediately or transferred to isolation rooms. The close contacts are given oseltamivir as $75 \mathrm{mg}$ capsules once daily for adults or $2 \mathrm{mg} / \mathrm{kg}$ (maximum of $75 \mathrm{mg}$ ) once daily for children for 3 days as PEP. All close contacts are monitored for development of influenza for 7 days after starting PEP.
\end{abstract}

Discussion: A 3-day regimen of oseltamivir as PEP has advantages over 7-to 10-day regimens in terms of costs, medication adherence and adverse effects.

Trial registration The Institutional Review Board of Hokkaido University Hospital for Clinical Research, 015-0518, registered on November 11, 2016. UMIN Clinical Trials Registry, UMIN000024458, disclosed on October 31, 2016. https://upload.umin.ac.jp/cgi-open-bin/ctr_e/ctr_view.cgi?recptno=R000027881. Japan Registry of Clinical Trials, jRCTs011180015, disclosed on March 14, 2019. https://jrct.niph.go.jp/latest-detail/jRCTs011180015

Keywords: Oseltamivir, Post-exposure prophylaxis, Influenza, Hospital wards

${ }^{*}$ Correspondence: nisgr@mrj.biglobe.ne.jp

${ }^{1}$ Division of Infection Control, Hokkaido University Hospital, Kita 14, Nishi

5, Kita-Ku, Sapporo, Hokkaido 060-8648, Japan

Full list of author information is available at the end of the article

\section{Background}

Post-exposure prophylaxis (PEP) using oseltamivir has been shown to be effective for reducing secondary infection of influenza in hospital wards [1]. A retrospective observational study showed that a 3-day regimen of oseltamivir as PEP for preventing transmission of influenza in wards is comparable to 7 - to 10 -day regimens 
provided index cases are immediately separated from close contacts [2]. In order to confirm the efficacy of a 3-day regimen of oseltamivir as PEP, we started to conduct a prospective, multi-center, single-arm trial.

\section{Methods/design}

\section{Aim}

The objective of this study is to investigate the efficacy of a 3-day regimen of oseltamivir for PEP of influenza in hospital wards.

\section{Study setting}

This study is a prospective, multi-center, single-arm study designed by the Sectional Meeting of Clinical Study, Japan Infection Prevention and Control Conference for National and Public University Hospitals in Japan. This trial will involve patients from Hokkaido University Hospital, Kagoshima University Hospital, Shinshu University Hospital, Tokyo Medical and Dental University Hospital, Tohoku University Hospital, Kobe University Hospital, Chiba University Hospital, Osaka City University Hospital, Tottori University Hospital and Shimane University Hospital in Japan.

\section{Endpoints}

The primary endpoint is the incidence of influenza for 7 days after starting PEP using oseltamivir by use of a 3-day regimen. The development of influenza is defined as development of influenza-like symptoms (e.g., fever, cough and runny nose) with a positive immunochromatographic test (ICT). The secondary endpoints are the associations of the incidence of influenza for 7 days after starting PEP using oseltamivir by use of a 3-day regimen with age, sex, influenza vaccination and underlying conditions of the patients because of the possibility of development of influenza in elderly patients, unvaccinated patients and immunocompromised patients even if they are prescribed oseltamivir.

\section{Study design}

The index patients with influenza are prescribed a neuraminidase inhibitor and are isolated from close contacts [2]. Close contacts who fulfill all of the criteria described in "Eligibility and exclusion" are given oseltamivir [75 mg capsules once daily for adults or $2 \mathrm{mg} / \mathrm{kg}$ (maximum of $75 \mathrm{mg}$ ) once daily for children] for 3 days as PEP. Since PEP with anti-influenza agents is widely practiced in Japan for both healthcare workers and patients, even for those who have already received vaccination, it is difficult to conduct a randomized controlled trial with comparison of a 3-day regimen of oseltamivir and a placebo control. The results of previous studies are destined to be used as historical controls [1-4].

\section{Eligibility and exclusion}

Eligible participants are (1) patients over 1 year of age, (2) patients sharing a room with index patients with influenza who are separated immediately (ex. by discharge or transferring to isolation rooms), (3) patients in whom administration of oseltamivir as PEP is started within $48 \mathrm{~h}$ after onset of fever of the index patients and (4) patients for whom written informed consent to participate in the study is obtained by the physicians participating in this study, from the patients themselves or from proxies. The following patients are not eligible for participation in this study: (1) patients with impairment of renal function (creatinine clearance $\leq 30 \mathrm{ml} / \mathrm{min}$ ) and (2) patients between 10 and 19 years of age, because the Japanese Ministry of Health, Labor and Welfare issued a warning against the use of oseltamivir in teenagers, except for those at high risks, after reports of two suicides by teenagers shortly after ingestion of oseltamivir in March 2007 [5].

\section{Study intervention and timeline of the study}

The following information will be obtained from each patient's record at the time of enrolment: (1) body temperature, respiratory symptoms (e.g., cough and runny nose) and results of an immunochromatographic test (ICT) of influenza in index patients, (2) clinical history including age, sex, body weight, influenza vaccination, past infection with influenza, underlying disease and medical history, and results of laboratory tests including hemoglobin, white blood count, aspartate aminotransferase (AST), alanine aminotransferase (ALT) and creatinine for the close contacts. Eligible participants for whom administration of oseltamivir is started as PEP are monitored for influenza-like symptoms (fever and respiratory symptoms) for 7 days (Table 1 ). If the eligible patients have influenza-like symptoms with a positive immunochromatographic test (ICT), they are regarded as having developed influenza. Information on adverse events will be obtained regardless of their grade of severity. This trial was declared and registered on November 11, 2016 (see "Trial status").

\section{Sample size}

In our previous study [2], we prophylactically administered oseltamivir for 3 days to 212 patients, and only 2 $(0.9 \%)$ of the patients developed influenza. In preceding clinical trials $[1,3,4]$, the proportion of patients with influenza infection in the placebo group was about $7 \%$. In this study, it is assumed that the proportion of patients with influenza will be lower than that in preceding studies due to the different study environments. Based on the idea of non-inferiority margin [6], the clinically 
Table 1 Schedule of data collection

\begin{tabular}{|c|c|c|c|c|c|c|c|c|}
\hline & \multirow[t]{2}{*}{ Before PEP ${ }^{a}$} & \multicolumn{7}{|c|}{ Observation period } \\
\hline & & Day 1 & Day 2 & Day 3 & Day 4 & Day 5 & Day 6 & Day 7 \\
\hline Informed consent & $x$ & & & & & & & \\
\hline Clinical assessment ${ }^{b}$ & $x$ & & & & & & & \\
\hline Laboratory test ${ }^{c}$ & $x$ & & & & & & & \\
\hline PEPa & & $x$ & $x$ & $x$ & & & & \\
\hline Fever & & $x$ & $x$ & $x$ & $x$ & $x$ & $x$ & $x$ \\
\hline Respiratory symptoms ${ }^{d}$ & & $x$ & $x$ & $x$ & $x$ & $x$ & $x$ & $x$ \\
\hline Immunochromatographic testing ${ }^{e}$ & & $x$ & $x$ & $x$ & $x$ & $x$ & $x$ & $x$ \\
\hline Adverse events & & $x$ & $x$ & $x$ & $x$ & $x$ & $x$ & $x$ \\
\hline
\end{tabular}

a Post-exposure prophylaxis

${ }^{b}$ Clinical assessment: history including age, sex, body weight, influenza vaccination, past infection with influenza, underlying disease and medical history

' Laboratory test: hemoglobin, white blood count, aspartate aminotransferase (AST), alanine aminotransferase (ALT) and creatinine

${ }^{d}$ Respiratory symptoms: cough or sneezing

${ }^{\mathrm{e}}$ If the close contacts with index patients with influenza have fever or respiratory symptoms within 7 days after PEP, immunochromatographic testing of influenza will be carried out

meaningful margin is set to be $50 \%$ of pre-existing risk difference $(\delta=7-1=6 \%)$. Therefore, the non-inferiority margin of our study is set to be $3 \%$. From the above argument, we assumed that the proportions of patients with influenza infection are $1 \%$ in this study (3-day regimen) and $4 \%$ in the null reference. Since the incidence of influenza in a hospital is relatively low and it is possible to confirm the presence or absence of influenza in a short period of time, a binomial distribution was assumed for the incidence. Using the binominal exact test statistic, the expected sample size was calculated to be 253 for detection of a $3 \%$ risk difference to achieve a power of $80 \%$ at a one-sided significance level of $2.5 \%$. Considering the dropout rate as $10 \%$, the final sample size became 279 .

\section{Statistical analysis}

For primary outcome assessment, a binomial (exact) test is performed to test the risk difference of $3 \%$ (proportions of patients with influenza infection being $4 \%$ for the null reference and $1 \%$ for the proposed regimen) at the onesided significance level of $2.5 \%$. Descriptive statistics are calculated for the secondary outcomes. All analyses are performed using SAS Version 9.4 (Cary, NC: SAS Institute Inc).

\section{Ethics}

The trial will be conducted under ICH E6 (R2) and Ethical Guidelines for Medical and Health Research Involving Human Subjects (Japan). The trial will adhere to the Clinical Trials Act (Japan) from April 1, 2019. Recruitment of participants can start following approval by the IRB of each hospital. The recruitment in Shinshu University
Hospital and Kobe University Hospital will be discontinued from April 1, 2019 onward.

\section{Data quality assurance}

The investigators will be responsible for implementing and maintaining quality assurance and quality control systems in accordance with written standard operating procedures. Centralized monitoring will be implemented in accordance with the manual prepared prior to the beginning of the trial.

\section{Data security}

Confidential patient information is kept secure by the rules of medical confidentiality and is treated according to the Act on the Protection of Personal Information in Japan.

\section{Safety}

Participants will be free to withdraw at any time from participation. An investigator may decide to terminate participation in the study if a participant meets an exclusion criterion (either newly developed or previously recognized) that precludes further participation.

\section{Adverse event (AE)}

An adverse event (AE) will be managed according to the FDA's guidance [7]. All severe adverse event (SAE) information will be reported to the IRB and must be shared with all investigators participating in the study. 


\section{Discussion}

The effectiveness of PEP using oseltamivir by use of 7 to 10-day regimens has been shown by several studies [1, $3,4,8]$. In a previous retrospective observational study conducted from the 2005/06 to 2014/05 influenza seasons, a 3-day regimen of oseltamivir as PEP for preventing transmission of influenza in wards was shown to be comparable to 7 - to 10-day regimens of oseltamivir provided index cases were immediately separated from close contacts [2]. A 3-day regimen of oseltamivir as PEP has advantages over 7 - to 10-day regimens as regards costs, medication adherence and adverse effects [9].

The incidence rate of influenza within a hospital and the expected incidence rate for the proposed regimen are both low; thus, for the detection of a small difference, the required sample size would become large to achieve nominal statistical power. In order to confirm the efficacy of a 3-day regimen of oseltamivir for PEP of influenza in wards, we started to conduct a prospective, multi-center, single-arm trial of a 3-day regimen of oseltamivir for PEP.

\section{Trial status}

This trial was declared and registered on November 11, 2016. Recruitment into the trial started on November 11, 2016 and will end in March 2024 or until a total of 279 participants have been recruited. Between November 2016 and May 2020, 68 patients were enrolled. Among them, 3 patients developed influenza after starting PEP using oseltamivir by use of a 3-day regimen.

\section{Abbreviations}

PEP: Post-exposure prophylaxis; ICH: International Conference on Harmonisation; AE: Adverse event; SAE: Serious adverse event.

\section{Acknowledgements}

We thank Stewart Chisholm for proofreading the manuscript.

\section{Authors' contributions}

$\mathrm{NI}$ conceived the study and wrote the first draft of the manuscript. MN, HK, SK, YN, KT, TM, HI, KY, HC, CS, RK, TY and NM participated in the design of the trial. YMI provided statistical expertise. SI is the qualitative researcher in the trial. All authors read and approved the final manuscript.

\section{Funding}

The authors have not received a specific grant for this research from any funding agency in the public, commercial or not-for-profit sectors. Oseltamivir is provided by each hospital participating in this study.

\section{Availability of data and materials}

Not applicable.

\section{Declarations}

Ethics approval and consent to participate

The study protocol was approved by the Institutional Review Board (IRB) of Hokkaido University Hospital for Clinical Research on November 11, 2016 (015-0518). The trial was disclosed at the UMIN Clinical Trials Registry
(UMIN000024458) on October 31, 2016 and at the Japan Registry of Clinical Trials (jRCTs011180015) on March 14, 2019. All participants will receive adequate information about the nature, purpose, possible risks and benefits of the trial and about alternative therapeutic choices using an informed consent approved by the IRB. Written informed consent will be obtained from a parent or proxy for a minor participant.

\section{Consent for publication}

Not applicable.

\section{Competing interests}

The authors declare that they have no competing interests.

\section{Author details}

${ }^{1}$ Division of Infection Control, Hokkaido University Hospital, Kita 14, Nishi 5, Kita-Ku, Sapporo, Hokkaido 060-8648, Japan. ${ }^{2}$ Department of Statistical Data Science, The Institute of Statistical Mathematics, 10-3 Midori-cho, Tachikawa, Tokyo 190-8562, Japan. ${ }^{3}$ Department of Infection Control and Prevention, Kyoto University Hospital, 54 Kawahara-cho, Shogoin, Sakyo-ku, Kyoto, Kyoto 606-8507, Japan. ${ }^{4}$ Department of Infection Control and Prevention, Division of Medical and Environmental Safety, Kagoshima University Hospital, 8-35-1 Sakuragaoka, Kagoshima, Kagoshima 890-8544, Japan. ${ }^{5}$ Division of Infection Control, Shinshu University Hospital, 3-1-1 Asahi, Matsumoto, Nagano 390-8621, Japan. ${ }^{6}$ Department of Infection Control and Prevention, Medical Hospital, Tokyo Medical and Dental University, 1-5-45 Yushima, Bunkyo-ku, Tokyo 113-8510, Japan. ${ }^{7}$ Department of Infection Control and Laboratory Diagnostics, Tohoku University Graduate School of Medicine, 2-1 Seiryo-machi, Aoba-ku, Sendai, Miyagi 980-8575, Japan. ${ }^{8}$ Infection Control Team, Kobe University Hospital, 7-5-2 Kusunoki-cho, Chuo-ku, Kobe, Hyogo 650-0017, Japan. ${ }^{9}$ Division of Infection Control, Chiba University Hospital, 1-8-1 Inohana, Chuo-ku, Chiba, Chiba 260-8677, Japan. ${ }^{10}$ Department of Infection Control

Science, Graduate School of Medicine, Osaka City University, 1-4-3 Asahimachi, Abeno-ku, Osaka, Osaka 545-8585, Japan. ${ }^{11}$ Center for Infectious Diseases, Tottori University Hospital, 36-1 Nishi-cho, Yonago, Tottori 683-8504, Japan. ${ }^{12}$ Division of Infection Control, Shimane University Hospital, 89-1 Enya-cho, Izumo, Shimane 693-8501, Japan. ${ }^{13}$ Department of Infectious Diseases, Nagoya University Hospital, 65 Tsurumai-cho, Showa-ku, Nagoya, Nagoya 466-8560, Japan. ${ }^{14}$ Center for Regional Medicine, Gifu University School of Medicine, 1-1 Yanagido, Gifu, Gifu 501-1194, Japan.

Received: 10 January 2020 Accepted: 23 August 2021

Published online: 30 August 2021

\section{References}

1. Shinjoh M, Takano Y, Takahashi T, Hasegawa N, Iwata S, Sugaya N. Postexposure prophylaxis for influenza in pediatric wards oseltamivir or zanamivir after rapid antigen detection. Pediatr Infect Dis J. 2012;31:1119-23.

2. Ishiguro N, Oyamada R, Nasuhara Y, Yamada T, Miyamoto T, Imai S, et al. Three-day regimen of oseltamivir for postexposure prophylaxis of influenza in wards. J Hosp Infect. 2016:94:150-3.

3. Welliver R, Monto AS, Carewicz O, Schatteman E, Hassman M, Hedrick J, et al. Effectiveness of oseltamivir in preventing influenza in household contacts: a randomized controlled trial. JAMA. 2001;285:748-54.

4. Hayden FG, Belshe R, Villanueva C, Lanno R, Hughes C, Small I, et al. Management of influenza in households: a prospective, randomized comparison of oseltamivir treatment with or without postexposure prophylaxis. J Infect Dis. 2004;189:440-9.

5. Okamoto E. Is oseltamivir (Tamiflu) safe? Re-examining the Tamiflu 'ado' from Japan. Expert Rev Pharmacoecon Outcomes Res. 2010;10:17-24.

6. Food Drug Administration Center for Drugs Evaluation Research. Guidance for industry: non-inferiority clinical trials to establish effectiveness. 2016. https://www.fda.gov/media/78504/download. Accessed 28 Jun 2020.

7. Food Drug Administration Center for Drugs Evaluation Research. Guidance for Clinical Investigators, Sponsors, and IRBs. 2009. https://www. fda.gov/media/72267/download. Accessed 14 Aug 2021. 
8. Chang YM, Li WC, Huang CT, Huang CG, Tsao KC, Cheng YH, et al. Use of oseltamivir during an outbreak of influenza $\mathrm{A}$ in a long-term care facility in Taiwan. J Hosp Infect. 2008;68:83-7.

9. Kato H, Hagihara M, Kato Y, Kurumiya A, Takahashi T, Sakata M, et al.

Adverse events of prophylactic anti-influenza agents in medical staffs. J Infect Chemother. 2017;23:683-6.

\section{Publisher's Note}

Springer Nature remains neutral with regard to jurisdictional claims in published maps and institutional affiliations.
Ready to submit your research? Choose BMC and benefit from:

- fast, convenient online submission

- thorough peer review by experienced researchers in your field

- rapid publication on acceptance

- support for research data, including large and complex data types

- gold Open Access which fosters wider collaboration and increased citations

- maximum visibility for your research: over 100M website views per year

At BMC, research is always in progress.

Learn more biomedcentral.com/submissions 